\title{
Prognostic Value of the Advanced Lung Cancer Inflammation Index in Patients with Lung Cancer: A Meta-Analysis
}

\author{
Yi Zhang $\mathbb{D}^{1}$ and Bo Chen ${ }^{2}$ \\ ${ }^{1}$ Department of General Surgery, The First People's Hospital of Neijiang, Neijiang, 641000 Sichuan Province, China \\ ${ }^{2}$ Department of Cardiology, The First People's Hospital of Neijiang, Neijiang, Sichuan Province, China \\ Correspondence should be addressed to Yi Zhang; 3455647525@qq.com
}

Received 29 March 2019; Accepted 6 May 2019; Published 1 July 2019

Academic Editor: Timo Sorsa

Copyright (c) 2019 Yi Zhang and Bo Chen. This is an open access article distributed under the Creative Commons Attribution License, which permits unrestricted use, distribution, and reproduction in any medium, provided the original work is properly cited.

\begin{abstract}
Background. The advanced lung cancer inflammation index (ALI) has been related to tumor survival in lung cancer (LC) patients. However, these findings regarding the prognostic relevance of ALI in LC were inconsistent. Our study is aimed at characterizing the prognostic significance of low pretreatment ALI in LC cases. Methods. Relevant published studies were systematically searched in several online databases. The combined hazard ratios (HRs) were applied to assess the correlation between ALI and overall/recurrence-free/progression-free survival (OS/PFS/RFS) in LC. Results. A total of 1587 LC patients from eight articles were recruited. Pooled results indicated that pretreatment ALI was significantly associated with prognosis in cases with LC. Compared to those with high-ALI, LC cases in the low-ALI group had a poorer OS (HR: 1.64, 95\% CI: 1.34-1.93, $p<0.001$ ). Subgroup analyses further revealed the negative significant prognostic value of low ALI in LC. In addition, low ALI had obvious connection with inferior PFS/RFS (HR: 1.71, 95\% CI: 1.35-2.07, $p<0.001$ ) in LC patients. Conclusions. Low ALI before treatments indicates poor prognosis in LC patients. Serum ALI may serve as a promising predictive tumor marker of survival in LC sufferers.
\end{abstract}

\section{Introduction}

Lung cancer (LC) is one of the most common and aggressive malignancies worldwide $[1,2]$. It is estimated that there are 234,030 new diagnosed cases of lung and bronchus cancer and 154,050 died from it in 2018 alone in the United States [1]. And LC ranked first in all of the cancer-related deaths in China $[3,4]$. In the pathological type, non-small-cell lung cancer (NSCLC) and small-cell lung cancer (SCLC) account for $85 \%$ and $15 \%$, respectively. Despite the development and improvements of diagnosis and treatments in LC, the prognosis of LC remains unsatisfactory, with a low longterm survival rate. It is of importance to find a novel index with an accurate predictive value in LC cases therefrom.

The advanced lung cancer inflammation index (ALI), as a newly reported inflammation-based prognostic score, is based on body mass index (BMI), serum albumin (ALB), and neutrophil to lymphocyte ratio (NLR). It was calculated as BMI $\times$ ALB/NLR [5]. The ALI was first evaluated as a powerful prognostic predictor in metastatic NSCLC [5], and subsequently, the usefulness of pretreatment ALI was evaluated as a prognostic marker in several types of malignancies, such as esophageal carcinoma and large B-cell lymphoma $[6,7]$. In recent years, the role of ALI as a promising biomarker in LC attracted wide attention; more cohort studies worked on the relationship between pretreatment ALI and survival in LC patients [8-14]. However, the results in these published studies remain inconclusive [8-14], and no meta-analysis concerning the prognostic value of low ALI in LC patients is available so far. Therefore, in this study, we synthetically examined the correlation between pretreatment ALI and prognosis in LC via summarizing all currently available data. We hypothesized that low ALI could be a candidate predictor that is valuable for predicting survival and progression in LC. 


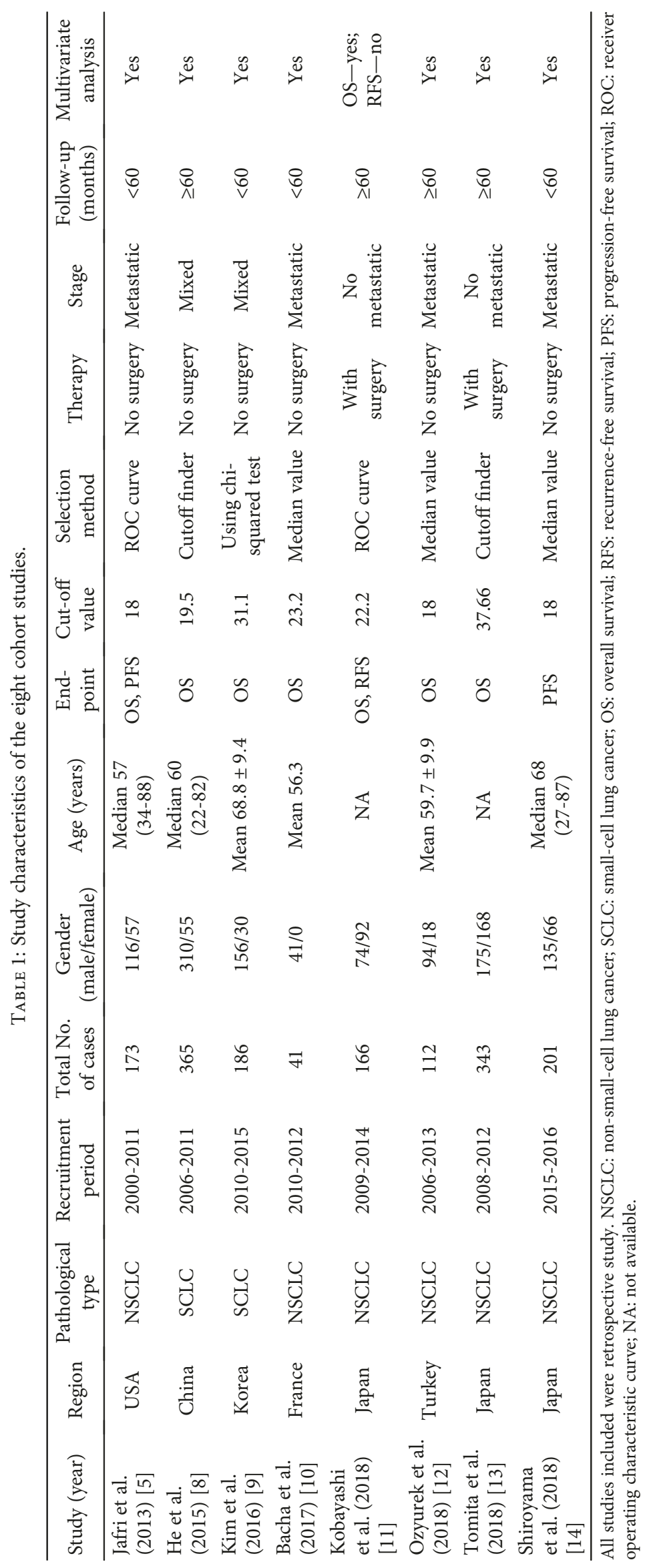




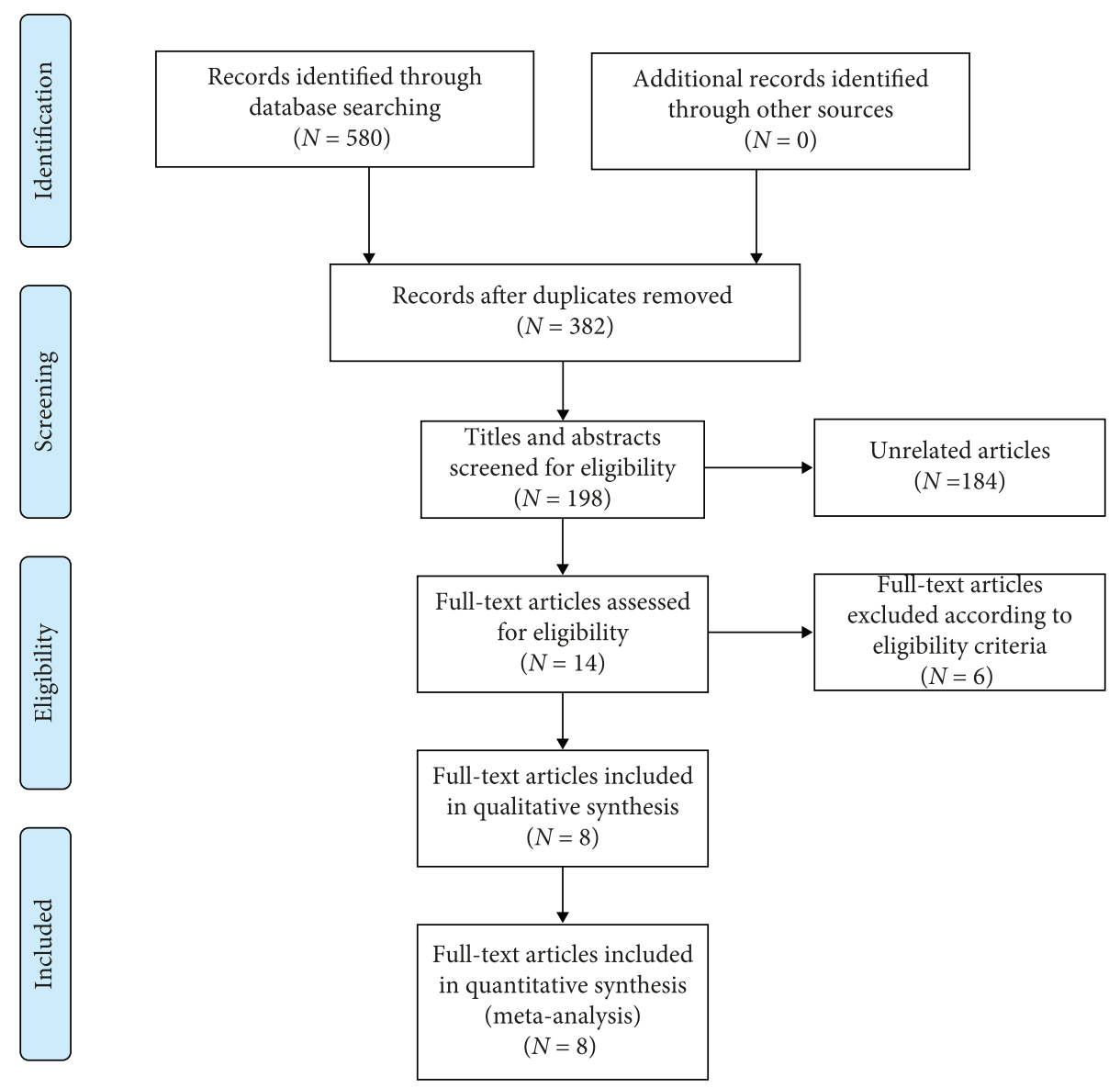

FIGURE 1: Flow diagram of included articles in the meta-analysis.

\section{Materials and Methods}

2.1. Search Strategy and Study Selection. PubMed, Web of Science, and Embase were systematically searched for potential studies. The following keywords and terms were used: "advanced lung cancer inflammation index," "ALI," "lung cancer," "lung tumor," or "lung carcinoma." The last update was Jan. 1, 2019, and no published language was restricted.

2.2. Inclusion and Exclusion Criteria. The inclusion criteria are as follows:

(1) All recruited subjects were pathologically diagnosed with primary LC

(2) The patients were divided into the low-ALI and highALI groups based on the pretreatment ALI levels

(3) The HRs for the overall survival (OS), progressionfree survival (PFS), or recurrence-free survival (RFS) were available

The exclusion criteria are as follows: reviews, abstracts, or posters or not involved in human lung cancer. And the latest study was included if there were overlapping data.
2.3. Data Extraction and Quality Assessment. Basic information of the included studies was extracted by two reviewers (Yi Zhang and Bo Chen), independently. The major features are listed in detail in Table 1. In addition, for the survival data, seven studies [5, 8-13] provided the HRs and 95\% CIs in multivariate analysis for OS, and the HRs in multivariate analysis for PFS were reported in two studies [5, 14]. Only one study reported the HRs for RFS in univariate analysis [11]. If a study considered patients with high ALI as the reference, then, the data was converted to HR estimations considering cases with low ALI as a reference group to reflect the impact of low ALI on LC participants. Quality assessment was assessed using the method that was described in detail by Lin et al. [15].

2.4. Statistical Analysis. All statistical analyses were carried out using Stata/SE14.1 (Stata Corp LP, College Station, Texas, USA). The associations between low ALI and OS or PFS/RFS in lung cancer were expressed as the hazard ratios (HRs) with their corresponding 95\% CIs. Heterogeneity across studies was evaluated by Cochran's $Q$ test and Higgins' $I^{2}$ statistic. The fixed effect model was adopted for nonsignificant heterogeneity $\left(I^{2}<50 \%, p>0.1\right)$. Publication bias was assessed by the visible plot and Begg's test, and sensitivity analysis was performed for the measurement of the reliability of the combined results. 


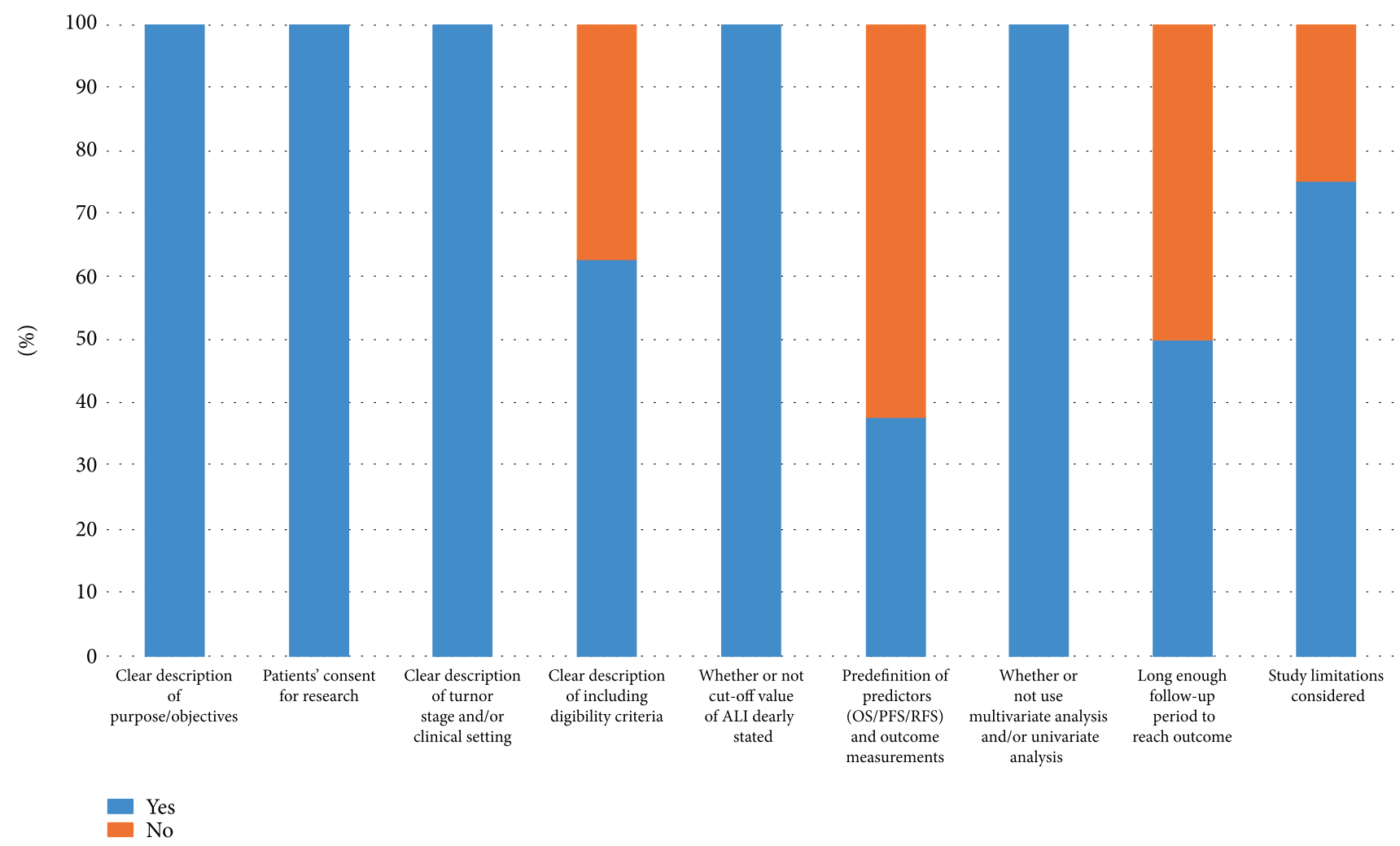

FIGURE 2: Quality assessment of 8 included studies.

\begin{tabular}{|c|c|c|c|}
\hline \multicolumn{2}{|l|}{$\begin{array}{l}\text { Study } \\
\text { ID }\end{array}$} & \multirow{2}{*}{$\frac{\operatorname{HR}(95 \% \mathrm{Cl})}{1.42(1.00,2.01)}$} & \multirow{2}{*}{$\begin{array}{c}\% \text { weight } \\
34.21\end{array}$} \\
\hline Jafri SH (2013) & - & & \\
\hline $\mathrm{He} \times(2015)$ & 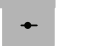 & $1.62(1.16,2.25)$ & 28.98 \\
\hline Kim EY (2016) & 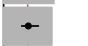 & $1.67(1.17,2.37)$ & 24.09 \\
\hline Bacha S (2017) & $\stackrel{1 \cdot}{+\cdot}$ & $2.38(1.05,5.18)$ & 2.03 \\
\hline Kobayashi S (2018) & 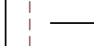 & $7.55(3.03,18.80)$ & 0.14 \\
\hline Ozyurek BA (2018) & $\rightarrow-$ & $1.83(0.89,3.79)$ & 4.13 \\
\hline Tomita M (2018) & $\rightarrow$ & $2.29(1.27,3.60)$ & 6.42 \\
\hline Overall $(I$-squared $=0.0 \%, p=0.586)$ & $\vartheta$ & $1.64(1.34,1.93)$ & 100.00 \\
\hline
\end{tabular}

FIgURe 3: Forest plot for the correlation between low-ALI and OS in LC.

\section{Results}

The detailed selection procedure was listed (Figure 1). According to the abovementioned criteria, finally, a total of eight studies [5,8-14] were considered to be eligible for this meta-analysis. There were altogether 1587 LC patients from eight retrospective studies with a mean sample size of 198.4. These studies were carried out in the USA (one study), China (one study), Korea (one study), France (one study), Japan (three studies), and Turkey (one study). Among them, seven studies reported OS, 2 studies covered PFS, and 1 study reported RFS. For the correlation between pretreatment ALI and OS, five studies worked on nonsmall-cell lung cancer (NSCLC), and two focused on small cell lung cancer (SCLC). The quality of the eight cohort studies was good with an average score of 7.25 (range 6-9; Figure 2, Table S1). The major characteristics of the recruited studies are summarized in Table 1.

3.1. ALI and OS. Seven cohort studies with a total of $1386 \mathrm{LC}$ patients reported the HRs for the association between pretreatment ALI and OS. The pooled analysis found that pretreatment ALI was closely linked to the prognosis of OS, and the LC patients with low-ALI had a shorter survival time (HR: 1.64, 95\% CI: 1.34-1.93, $p<0.001$ ) (Figure 3).

The prognostic values of low ALI in lung cancer were further displayed in subgroup analyses (Figures 4(a)-4(d), Table 2). Notably, low ALI could act as an adverse 


\begin{tabular}{|c|c|c|c|}
\hline \multicolumn{2}{|l|}{$\begin{array}{l}\text { Study } \\
\text { ID }\end{array}$} & \multirow[t]{2}{*}{$\mathrm{HR}(95 \% \mathrm{Cl})$} & \multirow[t]{2}{*}{$\%$ weight } \\
\hline NSCLC & 1 & & \\
\hline Jafri SH (2013) & 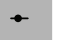 & $1.42(1.00,2.01)$ & 34.21 \\
\hline Bacha S (2017) & + & $2.38(1.05,5.18)$ & 2.03 \\
\hline Kobayashi S (2018) & - & $7.55(3.03,18.80)$ & 0.14 \\
\hline Ozyurek BA (2018) & ! & $1.83(0.89,3.79)$ & 4.13 \\
\hline Tomita M (2018) & $t_{\bullet}-$ & $2.29(1.27,3.60)$ & 6.42 \\
\hline Subtotal $\left(I^{2}=14.2 \%, p=0.324\right)$ & $\diamond$ & $1.64(1.21,2.07)$ & 46.93 \\
\hline SCLC & ! & & \\
\hline $\mathrm{He} \times(2015)$ & $\circ$ & $1.62(1.16,2.25)$ & 28.98 \\
\hline Kim EY (2016) & $\cdot$ & $1.67(1.17,2.37)$ & 24.09 \\
\hline Subtotal $(I$-squared $=0.0 \%, p=0.898)$ & $\diamond$ & $1.64(1.24,2.05)$ & 53.07 \\
\hline Heterogeneity between groups: $p=0.985$ ) & & & \\
\hline Overall $(I$-squared $=0.0 \%, p=0.586)$ & $\diamond$ & $1.64(1.34,1.93)$ & 100.00 \\
\hline
\end{tabular}

(a)

\begin{tabular}{|c|c|c|c|}
\hline \multicolumn{2}{|l|}{$\begin{array}{l}\text { Study } \\
\text { ID }\end{array}$} & \multirow[t]{2}{*}{$\mathrm{HR}(95 \% \mathrm{Cl})$} & \multirow[t]{2}{*}{$\%$ weight } \\
\hline Metastatic & $i$ & & \\
\hline Jafri SH (2013) & - & $1.42(1.00,2.01)$ & 34.21 \\
\hline Bacha S (2017) & $T \cdot-$ & $2.38(1.05,5.18)$ & 2.03 \\
\hline Ozyurek BA (2018) & $\vec{i}$ & $1.83(0.89,3.79)$ & 4.13 \\
\hline Subtotal $(I$-squared $=0.0 \%, p=0.609)$ & $\vartheta_{1}$ & $1.51(1.05,1.97)$ & 40.37 \\
\hline Mixed & & & \\
\hline $\mathrm{He} \times(2015)$ & - & $1.62(1.16,2.25)$ & 28.98 \\
\hline Kim EY (2016) & 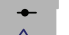 & $1.67(1.17,2.37)$ & 24.09 \\
\hline Subtotal $(I$-squared $=0.0 \%, p=0.898)$ & $\phi$ & $1.64(1.24,2.05)$ & 53.07 \\
\hline No-metastatic & & & \\
\hline Kobayashi S (2018) & - & $7.55(3.03,18.80)$ & 0.14 \\
\hline Tomita M (2018) & $\overrightarrow{0}$ & $2.29(1.27,3.60)$ & 6.42 \\
\hline Subtotal $(I$-squared $=40.2 \%, p=0.196)$ & P & $2.40(1.25,3.55)$ & 6.56 \\
\hline Heterogeneity between groups: $p=0.368$ ) & & & \\
\hline Overall $(I$-squared $=0.0 \%, p=0.586)$ & 8 & $1.64(1.34,1.93)$ & 100.00 \\
\hline
\end{tabular}

(b)

\begin{tabular}{|c|c|c|c|}
\hline \multicolumn{2}{|l|}{$\begin{array}{l}\text { Study } \\
\text { ID }\end{array}$} & \multirow[t]{2}{*}{$\mathrm{HR}(95 \% \mathrm{Cl})$} & \multirow[t]{2}{*}{$\%$ weight } \\
\hline No-surgery & 1 & & \\
\hline Jafri SH (2013) & - & $1.42(1.00,2.01)$ & 34.21 \\
\hline $\mathrm{He} \times(2015)$ & - & $1.62(1.16,2.25)$ & 28.98 \\
\hline Kim EY (2016) & - & $1.67(1.17,2.37)$ & 24.09 \\
\hline Bacha S (2017) & + & $2.38(1.05,5.18)$ & 2.03 \\
\hline Ozyurek BA (2018) & $\div$ & $1.83(0.89,3.79)$ & 4.13 \\
\hline Subtotal $(I$-squared $=0.0 \%, p=0.881)$ & $\diamond$ & $1.58(1.28,1.89)$ & 93.44 \\
\hline With-surgery & $i$ & & \\
\hline Kobayashi S (2018) & - & $7.55(3.03,18.80)$ & 0.14 \\
\hline Tomita M (2018) & 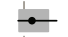 & $2.29(1.27,3.60)$ & 6.42 \\
\hline Subtotal $(I$-squared $=40.2 \%, p=0.196)$ & $\diamond$ & $2.40(1.25,3.55)$ & 6.56 \\
\hline Heterogeneity between groups: $p=0.177$ ) & & & \\
\hline Overall $(I$-squared $=0.0 \%, p=0.586)$ & $\diamond$ & $1.64(1.34,1.93)$ & 100.00 \\
\hline
\end{tabular}

(c)

Figure 4: Continued. 


\begin{tabular}{|c|c|c|c|}
\hline $\begin{array}{l}\text { Study } \\
\text { ID }\end{array}$ & & $\mathrm{HR}(95 \% \mathrm{Cl})$ & $\%$ weight \\
\hline$<60$ mon. & 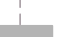 & & \\
\hline Jafri SH (2013) & $\rightarrow$ & $1.42(1.00,2.01)$ & 34.21 \\
\hline Kim EY (2016) & 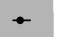 & $1.67(1.17,2.37)$ & 24.09 \\
\hline Bacha S (2017) & $T \cdot$ & $2.38(1.05,5.18)$ & 2.03 \\
\hline Subtotal $(I$-squared $=0.0 \%, p=0.597)$ & $\diamond$ & $1.55(1.17,1.93)$ & 60.33 \\
\hline$\geq 60$ mon. & i & & \\
\hline $\mathrm{He} \times(2015)$ & $\rightarrow$ & $1.62(1.16,2.25)$ & 28.98 \\
\hline Kobayashi S (2018) & - & $7.55(3.03,18.80)$ & 0.14 \\
\hline Ozyurek BA (2018) & $\rightarrow-$ & $1.83(0.89,3.79)$ & 4.13 \\
\hline Tomita M (2018) & $\rightarrow$ & $2.29(1.27,3.60)$ & 6.42 \\
\hline Subtotal $(I$-squared $=4.7 \%, p=0.369)$ & $\diamond$ & $1.77(1.30,2.24)$ & 39.67 \\
\hline Heterogeneity between groups: $p=0.479$ ) & 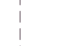 & & \\
\hline Overall $(I$-squared $=0.0 \%, p=0.586)$ & $\diamond$ & $1.64(1.34,1.93)$ & 100.00 \\
\hline
\end{tabular}

(d)

FIGURE 4: Forest plots for HRs of subgroup analyses for low ALI in LC.

TABLE 2: Subgroup analyses of the relationship between ALI and OS in LC patients.

\begin{tabular}{|c|c|c|c|c|c|}
\hline \multirow{2}{*}{ Subgroup factor } & \multirow{2}{*}{ No. of cohort studies } & \multirow{2}{*}{ Combined HR (95\% CI) } & \multirow{2}{*}{$p$ value } & \multicolumn{2}{|c|}{ Heterogeneity } \\
\hline & & & & $I^{2}(\%)$ & $P_{\text {het }}$ \\
\hline \multicolumn{6}{|l|}{ Pathological type } \\
\hline NSCLC & 5 & $1.64(1.21-2.07)$ & $<0.001$ & 14.2 & 0.329 \\
\hline SCLC & 2 & $1.64(1.24-2.05)$ & $<0.001$ & 0.0 & 0.898 \\
\hline \multicolumn{6}{|l|}{ Clinical stage } \\
\hline Metastatic & 3 & $1.51(1.05-1.97)$ & $<0.001$ & 0.0 & 0.609 \\
\hline Nonmetastatic & 2 & $1.64(1.24-2.05)$ & $<0.001$ & 0.0 & 0.898 \\
\hline Mixed & 2 & $2.40(1.25-3.55)$ & $<0.001$ & 40.2 & 0.196 \\
\hline \multicolumn{6}{|l|}{ Cut-off value } \\
\hline$<24.23$ & 5 & $1.57(1.21-1.92)$ & $<0.001$ & 0.0 & 0.510 \\
\hline$\geq 24.23$ & 2 & $1.80(1.27-2.33)$ & $<0.001$ & 0.0 & 0.351 \\
\hline \multicolumn{6}{|l|}{ Treatment } \\
\hline No surgery & 5 & $1.58(1.28-1.89)$ & $<0.001$ & 0.0 & 0.881 \\
\hline With surgery & 2 & $2.40(1.25-3.55)$ & $<0.001$ & 40.2 & 0.196 \\
\hline \multicolumn{6}{|l|}{ Follow-up } \\
\hline$<60 \mathrm{~m}$ & 3 & $1.55(1.17-1.93)$ & $<0.001$ & 0.0 & 0.597 \\
\hline$\geq 60 \mathrm{~m}$ & 4 & $1.77(1.30-2.24)$ & $<0.001$ & 4.7 & 0.369 \\
\hline
\end{tabular}

prognostic factor of OS in NSCLC (HR: 1.64, 95\% CI: 1.21-2.07, $p<0.001$ ) and SCLC (HR: 1.64, 95\% CI: 1.242.05, $p<0.001$ ). And the clinical stages (metastatic vs. mixed vs. no metastatic), cut-off value ( $\geq 24.23$ vs. $<24.23)$, treatment methods (no surgery vs. with surgery), and the follow-up time ( $\geq 60$ months vs. $<60$ months) all did not affect the significant predictive role of low-ALI in LC cases.

3.2. ALI and PFS/RFS. Three cohort studies with 540 LC subjects investigated the correlation between pretreatment ALI and PFS/RFS. The combined results showed pretreatment low ALI indicated worse PFS/RFS in LC (HR: 1.71,
95\% CI: $1.35-2.07, p<0.001)$, with no significant heterogeneity $\left(I^{2}=0.0 \%, p=0.965\right)$ (Figure 5 ).

3.3. Publication Bias. For the OS, the shape of Begg's funnel plot seems to be asymmetric $(\operatorname{Pr}>|z|=0.035)$ (Figure 6(a)), the estimated pooled result was still significant $(\mathrm{HR}=1.655$, 95\% CI: 1.402-1.952) after adjustment (Figure 6(b)) by the "trim-and-fill" method. For PFS/RFS, we did not conduct the publication bias assessment due to the small number of included studies.

3.4. Sensitivity Analysis. The sensitivity analysis indicated the results of our analyses were relatively stable in this metaanalysis (Figure 7). 


\begin{tabular}{|c|c|c|c|}
\hline $\begin{array}{l}\text { Study } \\
\text { ID }\end{array}$ & & $\mathrm{HR}(95 \% \mathrm{Cl})$ & $\%$ weight \\
\hline Jafri SH (2013) & $\longrightarrow$ & $1.66(1.18,2.33)$ & 39.62 \\
\hline Kobayashi S (2018) & & $1.79(1.11,2.65)$ & 22.10 \\
\hline Shiroyama T (2018) & & $1.72(1.24,2.41)$ & 38.28 \\
\hline Overall $(I$-squared $=0.0 \%, p=0.965)$ & & $1.71(1.35,2.07)$ & 100.00 \\
\hline
\end{tabular}

FIGURE 5: Forest plot for the correlation between low ALI and PFS/RFS in LC.

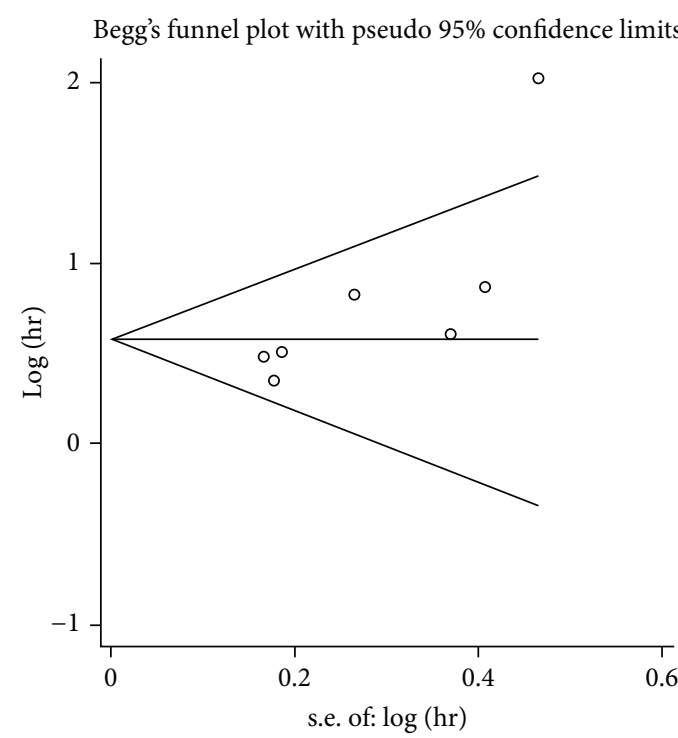

(a) Begg's funnel plot with pseudo 95\% confidence limits
Filled funnel plot with pseudo 95\% confidence limits

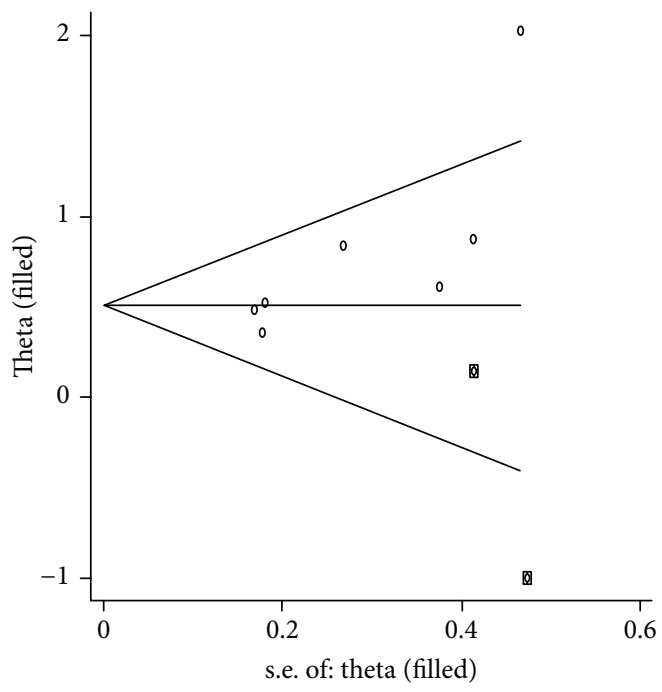

(b) Filled funnel plot with pseudo 95\% confidence limits

Figure 6: Publication bias assessment for OS: (a) Begg's funnel plot and (b) Filled funnel plot.

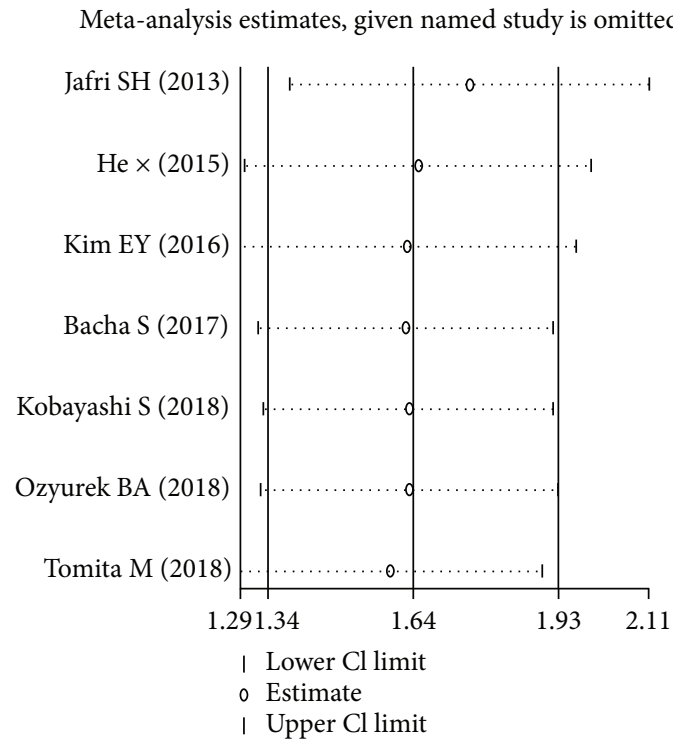

(a) Meta-analysis estimates; given named study is omitted

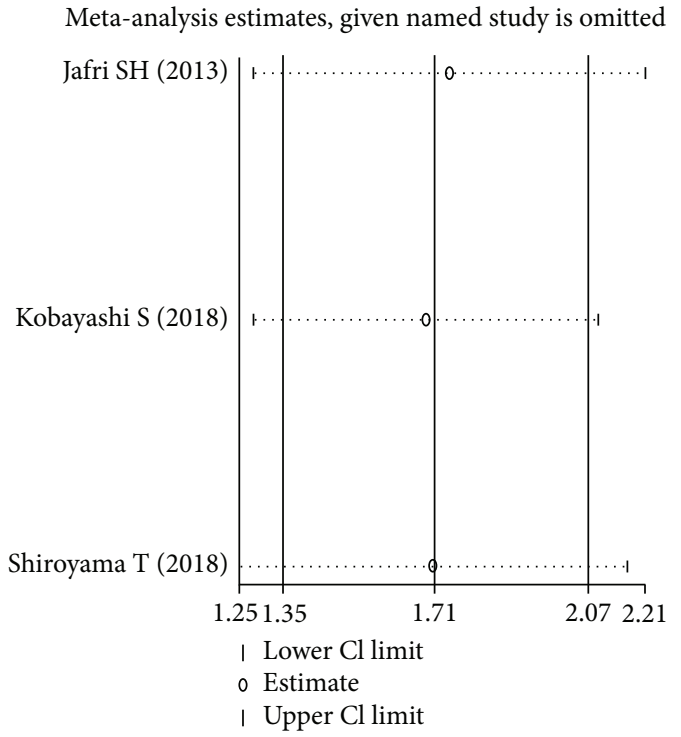

(b) Meta-analysis estimates; given named study is omitted

FIgURE 7: Sensitivity analysis for OS (a) and PFS/RFS (b). 


\section{Discussion}

In the current meta-analysis, a total of eight studies from six countries were collected for prognostic analysis. And to our knowledge, this is the first meta-analysis that focused on this field. Finally, there were altogether 1587 cases suffering from NSCLC or SCLC in this study. The combined results revealed a significantly shorter OS in LC cases with low ALI compared to those with high ALI, and low pretreatment ALI was also correlated with worse PFS/RFS in LC patients. Hence, these results suggested that a low ALI score was a promising indicator displaying negative prognostic values in LC patients.

The ALI is related to cancer survival and could be a prognostic indicator in cancer. However, the specific mechanism for the predictive value of this scoring system is uncertain. According to the definition, a low ALI based on a decreased BMI, a lower Alb, and/or a high NLR indicates poor prognosis and high systemic inflammation. BMI levels might represent the nutritional status of patients. Some studies reported that BMI could be a predictive factor of OS benefit in some tumors $[16,17]$. In addition, numerous studies have clarified a close association of inflammation and cancer [18-21]; a series of inflammatory cytokines could reflect inflammatory response and participate in the tumor development and progression [21, 22]. Among them, serum albumin was demonstrated as an indicator in cancers; hypoalbuminemia was closely associated with poor prognosis in many tumors [23-27]. In lung cancer, the low level of Alb was defined as a valuable index of poor survival rate and worse response [28, 29]. Furthermore, neutrophils and lymphocytes were recognized as two important factors in carcinogenesis [30, 31], and high NLR could be used as an independent negative marker of predicting prognosis in lung cancer [32, 33] and other malignancies, such as breast, colorectal, and esophageal cancers [34-36].

Aside from the limited studies available, the present meta-analysis had several other limitations. Firstly, the included cohort data were of a retrospective design. Secondly, negative data were usually difficult to be published. Thirdly, there was a possibility of publication bias for OS. Fourthly, the prognostic role of low ALI for other secondary outcomes such as PFS/RFS in LC needed further validation. Finally, there are variations in several aspects, such as the ALI measurement methods and the cutoff values among these studies. Considering the limitations listed above, multicenter researches with a better-designed and larger sample are required to further validate the clinical value of ALI in LC.

In conclusion, from our analyses of eight cohort studies, we concluded that LC patients with a low ALI score have poor survival outcomes. LC patients with low-serum ALI had a shorter OS and worse PFS/RFS. Therefore, pretreatment ALI may act as a valuable candidate with a predictive power in LC.
Abbreviations
ALI: Advanced lung cancer inflammation index
LC: $\quad$ Lung cancer
NSCLC: Non-small-cell lung cancer

SCLC: Small cell lung cancer

BMI: $\quad$ Body mass index

ALB: Albumin

NLR: Neutrophil to lymphocyte ratio

HRs: $\quad$ Hazard ratios

OS: Overall survival

RFS: $\quad$ Recurrence-free survival

PFS: Progression-free survival

ROC: Receiver operating characteristic curve

NA: Not available.

\section{Data Availability}

All the original data were available in the included published articles.

\section{Conflicts of Interest}

The authors have stated that they have no conflicts of interest.

\section{Supplementary Materials}

Table S1: study quality of eight studies in the meta-analysis. (Supplementary Materials)

\section{References}

[1] R. L. Siegel, K. D. Miller, and A. Jemal, "Cancer statistics, 2018," CA: A Cancer Journal for Clinicians, vol. 68, no. 1, pp. 7-30, 2018.

[2] L. A. Torre, R. L. Siegel, and A. Jemal, "Lung cancer statistics," Advances in Experimental Medicine and Biology, vol. 893, pp. 1-19, 2016.

[3] X. Zhang, L. Wu, Y. Xu et al., "Trends in the incidence rate of lung cancer by histological type and gender in Sichuan, China, 1995-2015: A single-center retrospective study," Thoracic Cancer, vol. 9, no. 5, pp. 532-541, 2018.

[4] J. Kong, F. Xu, M. He, K. Chen, and B. Qian, "The incidence of lung cancer by histological type: a population-based study in Tianjin, China during 1981-2005," Respirology, vol. 19, no. 8, pp. 1222-1228, 2014.

[5] S. H. Jafri, R. Shi, and G. Mills, "Advance lung cancer inflammation index (ALI) at diagnosis is a prognostic marker in patients with metastatic non-small cell lung cancer (NSCLC): a retrospective review," BMC Cancer, vol. 13, no. 1, p. 158, 2013.

[6] J. F. Feng, Y. Huang, and Q. X. Chen, "A new inflammation index is useful for patients with esophageal squamous cell carcinoma," OncoTargets and Therapy, vol. 7, pp. 1811-1815, 2014.

[7] Y. H. Park, H. G. Yi, M. H. Lee, C. S. Kim, and J. H. Lim, "Prognostic value of the pretreatment advanced lung cancer inflammation index (ALI) in diffuse large B cell lymphoma patients treated with R-CHOP chemotherapy," Acta Haematologica, vol. 137, no. 2, pp. 76-85, 2017.

[8] X. He, T. Zhou, Y. Yang et al., "Advanced lung cancer inflammation index, a new prognostic score, predicts outcome in patients with small-cell lung cancer," Clinical Lung Cancer, vol. 16, no. 6, pp. e165-e171, 2015. 
[9] E. Y. Kim, N. Kim, Y. S. Kim et al., "Prognostic significance of modified advanced lung cancer inflammation index (ALI) in patients with small cell lung cancer comparison with original ALI," PLoS One, vol. 11, no. 10, article e0164056, 2016.

[10] S. Bacha, A. Sghaier, S. Habibech et al., "Advanced lung cancer inflammation index: a prognostic score in patients with metastatic non-small cell lung cancer," La Tunisie Médicale, vol. 95, no. 11, pp. 976-981, 2017.

[11] S. Kobayashi, Y. Karube, T. Inoue et al., "Advanced lung cancer inflammation index predicts outcomes of patients with pathological stage IA lung adenocarcinoma following surgical resection," Annals of Thoracic and Cardiovascular Surgery, vol. 25, no. 2, pp. 87-94, 2019.

[12] B. A. Ozyurek, T. S. Ozdemirel, S. B. Ozden et al., "Does advanced lung inflammation index (ALI) have prognostic significance in metastatic non-small cell lung cancer?," The Clinical Respiratory Journal, vol. 12, no. 6, pp. 2013-2019, 2018.

[13] M. Tomita, T. Ayabe, and K. Nakamura, "The advanced lung cancer inflammation index is an independent prognostic factor after surgical resection in patients with non-small-cell lung cancer," Interactive Cardiovascular and Thoracic Surgery, vol. 26, no. 2, pp. 288-292, 2018.

[14] T. Shiroyama, H. Suzuki, M. Tamiya et al., "Pretreatment advanced lung cancer inflammation index(ALI) for predicting early progression in nivolumab-treated patients with advanced non-small cell lung cancer," Cancer Medicine, vol. 7, no. 1, pp. 13-20, 2018.

[15] Y. Lin, Z. Liu, Y. Qiu et al., “Clinical significance of plasma Ddimer and fibrinogen in digestive cancer: a systematic review and meta-analysis," European Journal of Surgical Oncology, vol. 44, no. 10, pp. 1494-1503, 2018.

[16] A. G. Renehan, M. Tyson, M. Egger, R. F. Heller, and M. Zwahlen, "Body-mass index and incidence of cancer: a systematic review and meta-analysis of prospective observational studies," The Lancet, vol. 371, no. 9612, pp. 569-578, 2008.

[17] J. E. Olson, P. Yang, K. Schmitz, R. A. Vierkant, J. R. Cerhan, and T. A. Sellers, "Differential association of body mass index and fat distribution with three major histologic types of lung cancer: evidence from a cohort of older women," American Journal of Epidemiology, vol. 156, no. 7, pp. 606-615, 2002.

[18] B. J. Rollins, "Inflammatory chemokines in cancer growth and progression," European Journal of Cancer, vol. 42, no. 6, pp. 760-767, 2006.

[19] K. Koizumi, S. Hojo, T. Akashi, K. Yasumoto, and I. Saiki, "Chemokine receptors in cancer metastasis and cancer cellderived chemokines in host immune response," Cancer Science, vol. 98, no. 11, pp. 1652-1658, 2007.

[20] T. N. Hartmann, M. Burger, and J. A. Burger, "The role of adhesion molecules and chemokine receptor CXCR4 (CD184) in small cell lung cancer," Journal of Biological Regulators and Homeostatic Agents, vol. 18, no. 2, pp. 126-130, 2004.

[21] L. M. Coussens and Z. Werb, "Inflammation and cancer," Nature, vol. 420, no. 6917, pp. 860-867, 2002.

[22] M. Sabzevary-Ghahfarokhi, H. Shirzad, M. Rafieian-Kopaei, M. Ghatreh-Samani, and M. Shohan, "The role of inflammatory cytokines in creating T cell exhaustion in cancer," Cancer Biotherapy \& Radiopharmaceuticals, vol. 33, no. 7, pp. 267273, 2018.
[23] X. Ouyang, Y. Dang, F. Zhang, and Q. Huang, "Low serum albumin correlates with poor survival in gastric cancer patients," Clinical Laboratory, vol. 64, 2018.

[24] J. Huang, Y. Wang, Y. Yuan et al., "Preoperative serum prealbumin as an independent prognostic indicator in patients with localized upper tract urothelial carcinoma after radical nephroureterectomy," Oncotarget, vol. 8, no. 22, pp. 3677236779, 2017.

[25] S. González-Trejo, J. F. Carrillo, D. D. Carmona-Herrera et al., "Baseline serum albumin and other common clinical markers are prognostic factors in colorectal carcinoma: a retrospective cohort study," Medicine, vol. 96, no. 15, article e6610, 2017.

[26] Y. X. Xu, Y. B. Wang, Y. L. Tan, C. Xi, and X. Z. Xu, "Prognostic value of pretreatment albumin to bilirubin ratio in patients with hepatocellular cancer: a meta-analysis," Medicine, vol. 98, no. 2, article e14027, 2019.

[27] H. Sun, P. Hu, H. Shen et al., "Albumin and neutrophil combined prognostic grade as a new prognostic factor in nonsmall cell lung cancer: results from a large consecutive cohort," PLoS One, vol. 10, no. 12, article e0144663, 2015.

[28] Y. Jin, L. Zhao, and F. Peng, "Prognostic impact of serum albumin levels on the recurrence of stage I non-small cell lung cancer," Clinics, vol. 68, no. 5, pp. 686-693, 2013.

[29] E. Espinosa, J. Feliu, P. Zamora et al., "Serum albumin and other prognostic factors related to response and survival in patients with advanced non-small cell lung cancer," Lung Cancer, vol. 12, no. 1-2, pp. 67-76, 1995.

[30] J. Á. Nicolás-Ávila, J. M. Adrover, and A. Hidalgo, "Neutrophils in homeostasis, immunity, and cancer," Immunity, vol. 46, no. 1, pp. 15-28, 2017.

[31] B. Farhood, M. Najafi, and K. Mortezaee, "CD8 ${ }^{+}$cytotoxic T lymphocytes in cancer immunotherapy: a review," Journal of Cellular Physiology, vol. 234, no. 6, pp. 8509-8521, 2018.

[32] S. Diem, S. Schmid, M. Krapf et al., "Neutrophil-to-lymphocyte ratio (NLR) and platelet-to-lymphocyte ratio (PLR) as prognostic markers in patients with non-small cell lung cancer (NSCLC) treated with nivolumab," Lung Cancer, vol. 111, pp. 176-181, 2017.

[33] S. J. Bagley, S. Kothari, C. Aggarwal et al., "Pretreatment neutrophil-to-lymphocyte ratio as a marker of outcomes in nivolumab-treated patients with advanced non-small-cell lung cancer," Lung Cancer, vol. 106, pp. 1-7, 2017.

[34] D. A. Patel, J. Xi, J. Luo et al., "Neutrophil-to-lymphocyte ratio as a predictor of survival in patients with triple-negative breast cancer," Breast Cancer Research and Treatment, vol. 174, no. 2, pp. 443-452, 2019.

[35] N. Dimitriou, E. Felekouras, I. Karavokyros, A. Alexandrou, E. Pikoulis, and J. Griniatsos, "Neutrophils to lymphocytes ratio as a useful prognosticator for stage II colorectal cancer patients," BMC Cancer, vol. 18, no. 1, p. 1202, 2018.

[36] Y. Sun and L. Zhang, "The clinical use of pretreatment NLR, PLR, and LMR in patients with esophageal squamous cell carcinoma: evidence from a meta-analysis," Cancer Management and Research, vol. 10, pp. 6167-6179, 2018. 


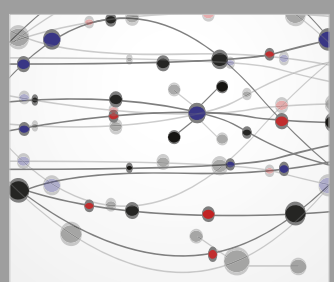

The Scientific World Journal
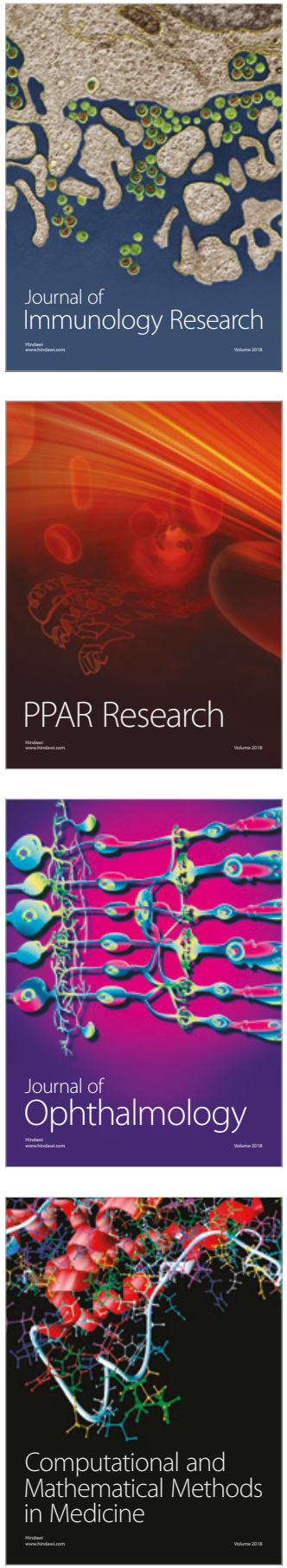

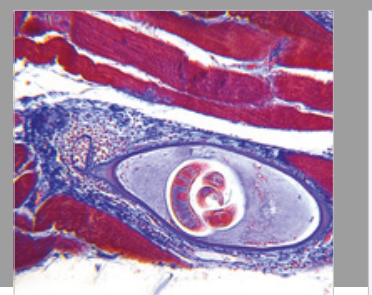

Gastroenterology Research and Practice

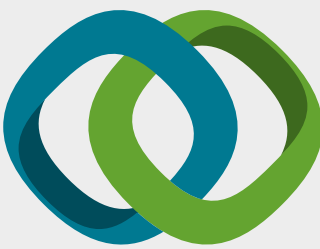

\section{Hindawi}

Submit your manuscripts at

www.hindawi.com
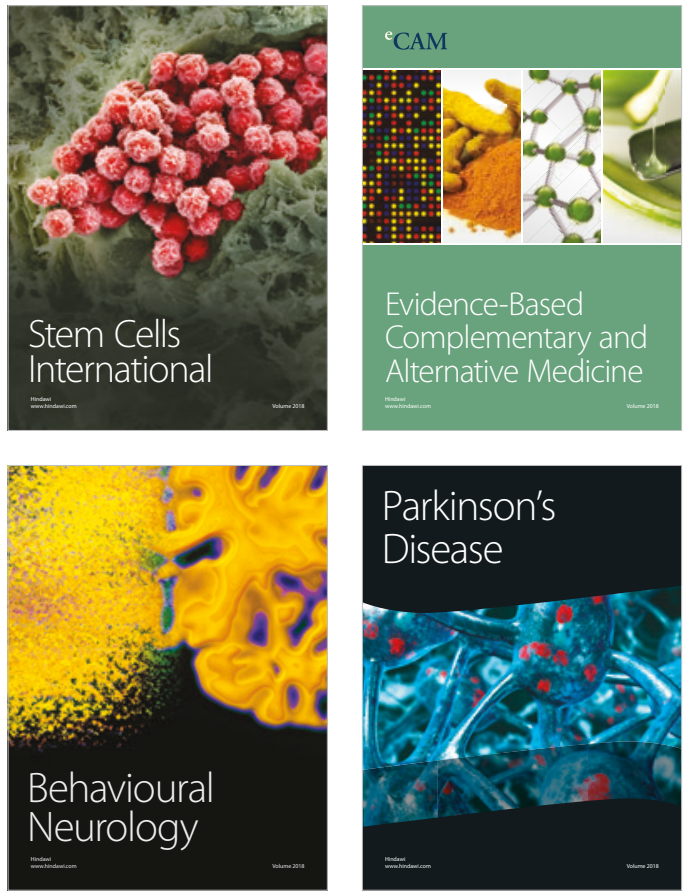

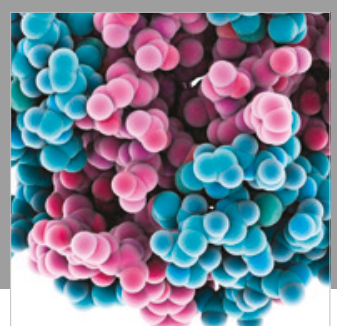

ournal of

Diabetes Research

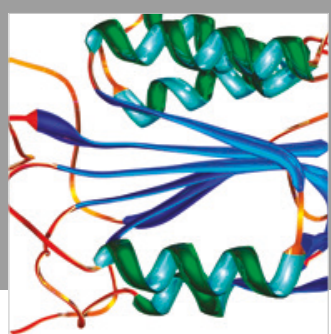

Disease Markers
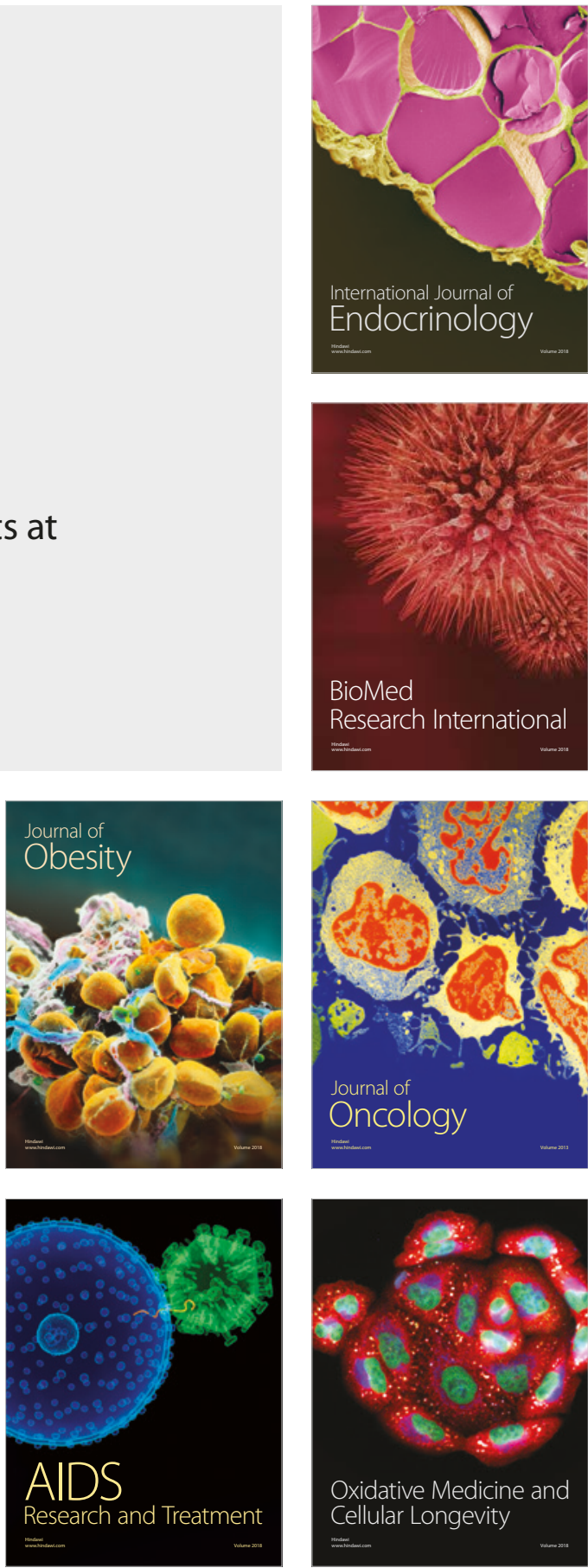